three gave unilateral shadows, the remaining two being radiologically negative. It seems reasonable to assume that, whatever the effect on the lungs, pain and tenderness of the chest muscles should occur. on the side facing the explosion; but in two of the five cases (Nos. 4 and 11) pain with or without tenderness was experienced on the side opposite to that described as facing the explosion. The remaining three cases had pain on the exposed side, the following case presenting the anomaly of muscle tenderness limited to the arm and chest facing the detonation, with fluid appearing in the opposite interlobar fissure.

\section{Case 10}

This patient was injured on Aug. 8, 1944, by a hand-grenade which struck his left shoulder and exploded on or close to him. He was unconscious for a short time. On coming round he noticed soreness of the left chest and shoulder, and was coughing bloody sputum. On examination there was obvious dyspnoea, with generalized rhonchi in both lungs. Chest radiologically clear.

Aug. 9.-Still coughing blood-stained sputum.

Aug. 11.-Cough and haemoptysis. Pain in left chest and arm. Radiographs gave an appearance of a small effusion in the right middle fissure.

Aug. 12.- No change radiologically.

Aug. 15.- Some pain in left chest on breathing. Occasional cough; mucoid sputum; no haemoptysis. Examination disclosed tenderness in the left pectoralis major and shoulder-girdle muscles. No abnormal signs in lungs. Radiographs revealed only a small amount of fluid in the right middle fissure.

It would be unwise to draw conclusions from the case histories, for the evidence seems contradictory in two instances: No. 4, whose history was of a land mine exploding on his left side, the subsequent muscular pain and tenderness being on the right; and No. 11, who gave a history of a mortar bomb exploding on the right and presented the anomalous combination of a recent perforation of the right drum, the left being intact, and pain and tenderness. of the left chest with radiological evidence of increased density of the left lower zone. Nor were any of the cases with unilateral histories and lesions comparable to Zuckerman's animals, for their chests were not protected on one side, and the blast may well have been received obliquely. Case 10 , however, would seem to suggest the possibility of a contrecoup lesion if it were not for the difficulty of accepting such an effect on an organ so elastic as the lung.

\section{Treatment}

Treatment should be for the symptoms. Hadfield (1940-1) has emphasized the importance of adequate rest. None of these patients developed pneumonia-a not uncommon complication according to O'Reilly (1941); many of those showing evidence of massive pulmonary haemorrhage had been given up to $20 \mathrm{~g}$. of a sulphonamide by the Army method of dosage of 5 tablets $\left(2 \frac{1}{2}\right.$ g.) twice a day during the first few days after injury, and this may well have prevented the complication. Physiotherapy would seem to be indicated for the muscular pain which is so prominent a symptom, followed by breathing exercises, but owing to pressure of more urgent work this could not always be carried out.

\section{Summary}

Twenty-one cases of blast injury to the lungs uncomplicated by other types of trauma are presented; the symptomatology, signs, and radiological findings are detailed. Haemoptysis as a persistent symptom is shown to bear no relation to the degree of pulmonary haemorrhage as manifested radiologically. Unilateral exposure to blast and the resulting lesions are found to have no constant relations; but in one case the possibility of contrecoup injury is mooted.

I would like to express my thanks to Dr. Geoffrey Doel for the $x$-raying of these patients and for his helpful co-operation at al times. I am also indebted to R.A.M.C. officers concerned for the early notes of the cases and in many instances for radiological investigations soon after injury.

\section{REFERENCES}

Dean, D. M., Thomas, A. R., and Allison, R. S. (1940). Lancet, 2, 224. Hadfield, G. (1940-1). Proc. roy. Soc. Med., 34, 189.

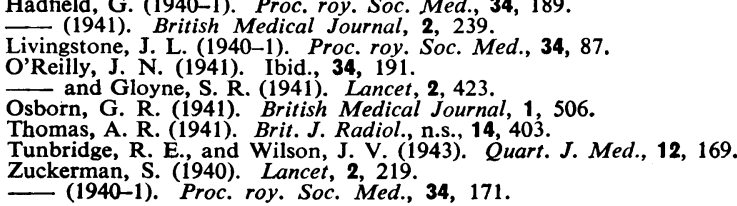

\section{AN OUTBREAK OF JAUNDICE WITH SIGNS IN THE NERVOUS SYSTEM}

\author{
E. A. J. BYRNE, M.D., M.R.C.P. \\ Major, R.A.M.C. \\ AND
}

\section{G. F. TAYLOR, M.R.C.P.}

Lieut.-Col., I.M.S.

In the steadily growing literature on infective hepatitis there is scant reference to lesions in the nervous system. In a series of 170 cases studied by Cameron (1943) the only neurological sign noted was a temporary paresis of accommodation in two cases. Newman (1942) refers to severe headache and to the occasional occurrence of paraesthesiae in the limbs. In none of his cases was the cerebrospinal fluid examined. Brain (1943) refers to one case in which unilateral convulsions and a hemiplegia, together with mild polyneuritic signs, preceded the onset of jaundice. Lescher (1944) gives three references to single cases of meningitis associated with infective hepatitis and describes two cases. A housewife aged 28 during an epidemic of infective hepatitis developed signs of a generalized polyneuritis which was followed one week later by jaundice ; recovery was complete. In the second case an R.A.S.C. driver suddenly developed a left hemiplegia two months after an attack of epidemic hepatitis, from which he had made a complete recovery; there seems little justification for associating these two illnesses.

\section{History of the Outbreak}

The cases which are the subject of this note show the association of lesions of the nervous system with jaundice in several individuals living in close proximity. In Sept., 1943, a casualty clearing station in the Arakan, on the Burma front, was receiving a few cases of infective hepatitis each week, differing in no way from the usual type. Within two days 5 cases of jaurndice with unusual neurological symptoms and signs were admitted. Four of these patients were from one British regiment and the fifth was an officer who had been working with this unit.

The history and clinical findings in four of the cases were very similar. The onset of the illness was sudden, occurring while the battalion was marching down to relieve another in the front line. All four men went sick within 48 hours of each other, with severe headache, dizziness, and a rise in temperature to about $104^{\circ}$ F. Pain behind the eyes and generalized body pains, worse in the neck and in the back, were constant features. A " pins and needles" or tingling sensation rapidly developed in the arms and legs, with loss of muscular power, particularly in the lower limbs. Some degree of dimness of vision was noticed by each of the patients.

The fever lasted two or three days. A moderate jaundice developed on about the fourth day. When examined at the C.C.S. 10 days after the onset the following signs were noted. All four men were mildly jaundiced and bile was present in the urine. The liver was tender and enlarged and the spleen was palpable in all cases. The total and differential white counts were normal. The men complained of dizziness, dimness of vision, unsteadiness, and weakness of the limbs. On attempting to walk they staggered. During the first few days of the illness Kernig's sign had been reported as positive; a slight degree of neck rigidity still remained. The fundi were normal apart from a fullness of the veins in three cases. The remaining cranial nerves were normal. There was no loss of power, and no incoordination was present in the upper limbs. The arm and abdominal reflexes were normal. The lower limbs showed varying degrees of muscular weakness. The knee-jerks were diminished, though unequally on the two sides; in one case the right knee-jerk was absent. The ankle-jerks were absent. The plantar responses were flexor, except in one case which showed equivocal responses. In two cases there was definite sensory loss in the thigh - in one case on the anterior surface and in the other along the posterior surface. The cerebrospinal fluid was abnormal in three of the cases. In two a clot developed 15 minutes after the fluid had been withdrawn. The protein content was increased in three cases. Cell counts were 23, 14, and 11 lymphocytes per c.mm. The Wassermann reaction was 
negative in all the fluids. Sera of these cases gave negative results on agglutination with the leptospira of Weil's disease.

The patients were evacuated to base hospitals, where three of them made a complete recovery within two months. The other two cases developed sequelae, as described below.

\section{Case IV}

C.S.M. J., aged 32, was seen by Major A. D. Leigh, R.A.M.C., neurologist, in Dec., 1943, three months after the onset. The following notes are extracted from his report. The patient complained of weakness and numbness in the legs. There was no jaundice and the liver was not palpable. The pupils and cranial nerves were normal. There was slight weakness of both hand-grips, with exaggerated reflexes in both limbs. Blunting to cotton-wool and pinprick was present on both hands. Power in both lower limbs was diminished, more particularly in the right leg. Muscle tone was increased in both legs and the gait was mildly spastic. The deep reflexes were much exaggerated, except the right ankle-jerk, which was diminished. The plantar responses were flexor. There was diminished sensation on the outer side of both thighs and over the dorsum of the feet, with absolute loss on the toes. The cerebrospinal fluid was normal. The patient was invalided to the U.K.

\section{Case V}

On Sept. 2, 1943, Capt. R., aged 33, complained of sore throat. On the 9th he had a rigor, which recurred despite quinine. On Sept. 14 he was admitted to an M.D.S., where his urine was noted to be dark in colour. The next day he complained of pains in the calves and thighs, and these were followed by loss of power from the hips downwards. On Sept. 17 there was a complete flaccid paraplegia, with anaesthesia below the groins. The patient complained of pain in the right arm, and on examination weakness of abduction was noted. There was no complaint of headache. On Sept. 19 he was admitted to a C.C.S.; he vomited several times that day. The next day he complained of headache, pain in the back, and blurring of vision with the onset of fever. There was difficulty in emptying the bladder. Jaundice was now present and the liver was enlarged and tender. The C.S.F. showed no increase in cells and the protein content was normal. The white cells numbered 9,000 per c.mm. Improvement was noted during the next few days, and on Oct. 2, on arrival at a field hospital, a considerable return of power and sensation had taken place in the left lower limb and, to a less degree, in the right. By the end of October no weakness of the right arm could be detected. The abdominal reflexes were normal. There was weakness of flexion of the right knee and of dorsiflexion of the right foot. Tone was increased in the right leg, particularly in the tendo Achillis. There was no appreciable wasting, and sensation was normal. The deep reflexes were exaggerated in the lower limbs, more particularly on the right side. There was unsustained right-sided ankle clonus, and the right plantar response was equivocal, while the left was flexor. By the middle of November power had further returned in the right leg, and the plantar response was now flexor. The patient was evacuated to the U.K.

\section{Discussion}

In four of the five cases described above an abrupt onset of fever, generalized pains, and signs of a mild meningitis were quickly followed by blurring of vision, weakness, and paraesthesiae in the lower limbs. In the fourth case pyramidal signs were subsequently noted, while in the fifth case the signs were predominantly those of a myelitis. Jaundice associated with enlargement of the liver and spleen appeared in all cases between the 4 th and 6 th days of the illness. Recovery was rapid and complete in three of them. In the remaining two a mild residual spastic paresis of the lower limbs, with in one case sensory loss peripherally, was still present several months later.

In considering the aetiology of the nervous signs in these cases acute poliomyelitis need be considered only in Case V. In Sept., 1943, only a single case of this disease was reported from this part of the Burma front. Furthermore, the myelitic form is distinctly rare, and it is unusual in such cases for recovery to be so rapid and the sequelae so mild. The normal cerebrospinal findings on the fifth day of the disease are also unusual. Since another case with mild myelitic signs occurred in the series, and since there were features in Case $\mathrm{V}$ rarely seen in acute poliomyelitis, this has been included.

The jaundice appeared to be of the usual infective type, especially since other cases from the same area were seen at that time. Assuming that these men suffered from infective jaundice, the question of neurotropism of the virus causing this condition must be considered. It is known that, experimentally, hepatotropic and neurotropic elements may coexist in viruses-e.g., yellow fever. On the other hand, taking into consideration the widespread distribution of infective hepatitis during the present world war and the extreme rarity of nervous complications, it is doubtful whether neurotropism of this virus can be accepted as the explanation in these cases. There remains the coincidental association of two diseases-a not uncommon event in the East. Encephalomyelitis, to which one case bears some resemblance, although not uncommon in peacetime, has been rare in the Army in India during the present war. We have not attempted to give a label to the neurological picture which these cases presented, but have indicated that meninges, spinal cord, and peripheral nerves were affected in varying degree.

\section{Summary}

A description is given of a small outbreak on the Burma front of a condition characterized by the association of jaundice with meningitis, peripheral neuritis, and, less constantly, myelitis.

The relationship of these conditions is briefly discussed.

Our thanks are due to Lieut.-Gen. W. Wilson, C.B., C.B.E., K.H.S., Director of Medical Services for India, for permission to publish this paper.

\section{REFERENCES} Cameron. J. D. S. (1943). Ouart. J. Med., 36, 139. Lescher, F. G. (1944). British Medical Journal, 1, 554 Newman, J. L. (1942). Ibid., 1, 61 .

\section{A SOCIAL STUDY OF HOSPITAL TREATMENT} BY

MALCOLM BROWN, D.Phil., M.R.C.P.

AND

FREDA C. CARLING, A.I.H.Á.

(From the Nuffield Department of Clinical Medicine, Radcliffe Infirmary, Oxford)

Anyone with experience of modern hospital work knows that facilities for in-patient treatment are limited. It is therefore important that they should be used to the best advantage. Also, all those who deal with the sick know something of the impact on family life made by a serious illness, but few realize how it is borne. The present study is an attempt to assess the value and efficiency of the in-patient service of a general medical ward, and to discover how such a misfortune as a hospital illness is met by the patient.

\section{The Patients Studied}

Contact was made with all the patients who had been admitted to Collier Ward during a period of six months to see how far medical and social care had been adequate to meet their needs. The period between Aug. 1, 1941, and Jan. 31, 1942, was chosen because during that time one of us (M.B.) had been housephysician.

Collier Ward is divided into accommodation for 10 men and 11 women. It is attached to the Nuffield Department of Clinical Medicine, and although when it was opened in 1938 it was mainly intended for scientific investigation and research, by 1941 it was also taking its share in the clinical teaching of students and the general work of the hospital. The ward was staffed with a sister, seven nurses, and a house-physician, and the bed costs for the six months under review were $£ 2,280$.

The follow-up was begun in Feb., 1943, and a letter was ent to each of the 156 patients explaining the nature of the investigation and asking for their co-operation. Wherever possible the social worker (F.C.C.) then visited the patients, discussed the social implications of their illness, and arranged for them to come up to hospital for a medical examination. Seventy-two patients were visited in their own homes; 51 attended for medical examination; 31 lived too far away for a visit, but sent a letter and report from their own doctor. Only 6 remained untraced, and of these one was an evacuee, one a tramp, and two were from a near-by construction camp. Notes were made on the medical and social aspects of each case, and when the record was complete the questionary shown in Table I was filled in. All but two of the patients were delighted to hear from the hospital and know the staff were interested in their progress. They gave us unlimited co-operation. 